\title{
Skyrmion formation in a bulk chiral magnet at zero magnetic field and above room temperature
}

\author{
K. Karube, ${ }^{1}$ J. S. White, ${ }^{2}$ D. Morikawa, ${ }^{1}$ M. Bartkowiak, ${ }^{3}$ A. Kikkawa, ${ }^{1}$ Y. Tokunaga, ${ }^{4}$ T. Arima, ${ }^{1,4}$ H. M. Rønnow, ${ }^{5}$ \\ Y. Tokura, ${ }^{1,6}$ and Y. Taguchi ${ }^{1}$ \\ ${ }^{1}$ RIKEN Center for Emergent Matter Science (CEMS), Wako 351-0198, Japan \\ ${ }^{2}$ Laboratory for Neutron Scattering and Imaging (LNS), Paul Scherrer Institute (PSI), CH-5232 Villigen, Switzerland \\ ${ }^{3}$ Laboratory for Scientific Developments and Novel Materials (LDM), Paul Scherrer Institute (PSI), CH-5232 Villigen, Switzerland \\ ${ }^{4}$ Department of Advanced Materials Science, University of Tokyo, Kashiwa 277-8561, Japan \\ ${ }^{5}$ Laboratory for Quantum Magnetism (LQM), Institute of Physics, École Polytechnique Fédérale de Lausanne (EPFL), \\ CH-1015 Lausanne, Switzerland \\ ${ }^{6}$ Department of Applied Physics, University of Tokyo, Bunkyo-ku 113-8656, Japan
}

(Received 26 June 2017; published 15 December 2017; corrected 28 December 2018)

\begin{abstract}
We report that in a $\beta$-Mn-type chiral magnet $\mathrm{Co}_{9} \mathrm{Zn}_{9} \mathrm{Mn}_{2}$, skyrmions are realized as a metastable state over a wide temperature range, including room temperature, via field cooling through the thermodynamic equilibrium skyrmion phase that exists below a transition temperature $T_{\mathrm{c}} \sim 400 \mathrm{~K}$. The once-created metastable skyrmions survive at zero magnetic field both at and above room temperature. Such robust skyrmions in a wide temperature and magnetic field region demonstrate the key role of topology and provide a significant step toward technological applications of skyrmions in bulk chiral magnets.
\end{abstract}

DOI: 10.1103/PhysRevMaterials.1.074405

\section{INTRODUCTION}

Topological states are in general endowed with robustness against disturbances, such as quenched disorder and thermal agitation, since they cannot be converted into a state with different topology through a continuous deformation process. A magnetic skyrmion, a vortexlike spin swirling structure, is one such example of a topological object in magnets and can be viewed as a particle which is characterized by an integer termed the skyrmion number [1-4]. Recent extensive studies have clarified that skyrmions are indeed stable to some extent due to their topological nature and exhibit a rich variety of emergent phenomena. In addition, they can be manipulated with a current density of $\sim 10^{6} \mathrm{~A} \mathrm{~m}^{-2}$, which is five to six orders of magnitude smaller than that required to drive ferromagnetic domain walls, making them promising for spintronics applications [5-9]. Various kinds of magnets have been found to host skyrmions [3,4,10-20], and their origins are attributed to several microscopic mechanisms.

One representative mechanism is a competition between a ferromagnetic exchange interaction and a DzyaloshinskiiMoriya interaction (DMI), the latter of which arises from broken inversion symmetry either at heterointerfaces [1013,21,22] or in bulk materials [3,4,15-20] with chiral or polar structures. For example, the interfacial DMI gives rise to skyrmions in ferromagnetic ultrathin multilayer films at low temperatures $[10,11]$ and even at room temperature $[12,13]$. On the other hand, skyrmions in bulk materials have been investigated in structurally chiral magnets such as B20-type compounds (e.g., $\mathrm{MnSi}[3], \mathrm{Fe}_{1-x} \mathrm{Co}_{x} \mathrm{Si}[4,16]$, and FeGe [17]) and $\mathrm{Cu}_{2} \mathrm{OSeO}_{3}$ [18,19]. In zero magnetic field, these compounds exhibit a long-period helical magnetic state, described by a single magnetic modulation vector ( $q$-vector), as a ground state. Slightly below the helimagnetic transition temperature $T_{\mathrm{c}}$, application of a magnetic field induces the topological transition to a triangular-lattice skyrmion crystal (SkX) state [Fig. 1(a), left], where the magnetic lattice is often described in terms of triple $q$-vectors that are rotated by $120^{\circ}$ with respect to each other and perpendicular to the magnetic field. In bulk, the thermodynamical equilibrium SkX state is confined to a narrow temperature and magnetic field region just below $T_{\mathrm{c}}$, and helical or conical states with zero topological charge are the thermodynamically most stable states at lower temperatures. However, it is necessary to create skyrmions in a wider temperature and magnetic field region including room temperature and zero magnetic field for both fundamental investigations and practical applications.

Recently, skyrmions have been found as an equilibrium state at and above room temperature in bulk Co-Zn-Mn alloys [20] with the $\beta$-Mn-type chiral crystal structure (space group $P 4_{1} 32$ or $P 4_{3} 32$ ) [see the inset of Fig. 2(a)] [23,24]. Mn-free $\mathrm{Co}_{10} \mathrm{Zn}_{10}$ hosts a helical state below $T_{\mathrm{c}} \sim 460 \mathrm{~K}$, and $T_{\mathrm{c}}$ decreases as the partial substitution with $\mathrm{Mn}$ proceeds. In $\mathrm{Co}_{8} \mathrm{Zn}_{8} \mathrm{Mn}_{4}\left(T_{\mathrm{c}} \sim 300 \mathrm{~K}\right)$, a triangular-lattice SkX appears as an equilibrium state around $290 \mathrm{~K}$ under a magnetic field. Although the temperature is around room temperature, this equilibrium phase exists only in a narrow temperature and magnetic field region similar to the other materials. On the other hand, it was found that a supercooled metastable SkX state is realized by field-cooling (FC) with a moderate rate of $\sim 1 \mathrm{~K} \mathrm{~min}^{-1}$. Once created, the metastable SkX survives over a wide temperature region below $280 \mathrm{~K}$ (including at zero field) [25], and the SkX coordination transforms into a squarelike one [Fig. 1(a), right] at low temperatures in the metastable state $[25,26]$. Despite these advances, skyrmions prevailing in a wide range of temperature and magnetic field, including above room temperature and zero field, remain to be demonstrated in bulk chiral magnets. In addition, a microscopic mechanism for the robustness of metastable skyrmions has remained elusive. To shed light on these problems, in the present study, we focused on $\mathrm{Co}_{9} \mathrm{Zn}_{9} \mathrm{Mn}_{2}$ with presumably similar properties, but a much higher $T_{\mathrm{c}} \sim 400 \mathrm{~K}$, and performed small-angle neutron scattering (SANS) and ac susceptibility measurements on a bulk sample, and Lorentz transmission electron microscopy (LTEM) measurements on a thin-plate specimen. As summarized in the state diagram in Fig. 1(b), we 

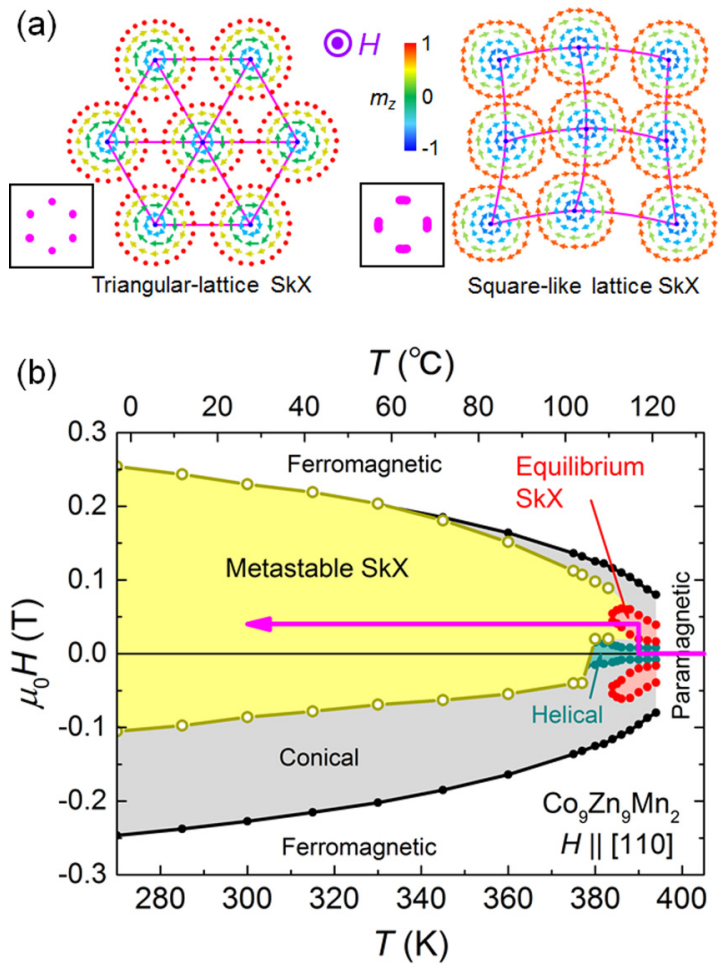

FIG. 1. (a) Schematic figures of a triangular-lattice SkX and a squarelike-lattice $\mathrm{SkX}$ in real space. Magnetic moments are indicated by arrows, and their $z$ components $\left(m_{z}\right)$ parallel to the magnetic field $(H)$ are represented by colors. The corresponding reciprocal space (SANS) patterns are also indicated in the boxes on the left-hand sides of the real space configurations. (b) Temperature ( $T$ )- $H$ state diagram of bulk $\mathrm{Co}_{9} \mathrm{Zn}_{9} \mathrm{Mn}_{2}$. The phase boundaries of helical multidomain (dark green area), thermodynamical equilibrium $\mathrm{SkX}$ (red area), conical (gray area), and induced-ferromagnetic states (white area below $T_{\mathrm{c}} \sim 396 \mathrm{~K}$ ), all indicated with solid symbols, are determined by isothermal ac susceptibility measurements [see Fig. 3(c)] in field-increasing runs after zero field cooling (ZFC) from $400 \mathrm{~K}$ to the measurement temperature. The boundaries of the metastable SkX state (yellow area) denoted with open symbols are determined from isothermal ac susceptibility measurements [see Fig. 8(d)] done as field-sweeping runs from $0.04 \mathrm{~T}$ to $\pm 0.3 \mathrm{~T}$ after field cooling (FC) via the equilibrium SkX phase as described by the pink arrow. Note that the displayed metastable SkX state was originally created in the positive-field equilibrium phase and persists down to the negative-field region below $380 \mathrm{~K}$, where the transition to helical state is prevented.

have succeeded in observing a highly robust metastable SkX for a wide temperature and magnetic field range including room temperature and zero magnetic field in both the bulk and thin-plate samples.

\section{EXPERIMENTAL METHODS}

\section{A. Sample preparation}

An ingot of $\mathrm{Co}_{9} \mathrm{Zn}_{9} \mathrm{Mn}_{2}$ composed of several singlecrystalline grains was grown by the Bridgman method as reported elsewhere $[20,25]$. Phase purity with a $\beta$-Mn-type crystal structure was confirmed by $\mathrm{x}$-ray diffraction on a powder obtained by crushing pieces from the same batch of (a)

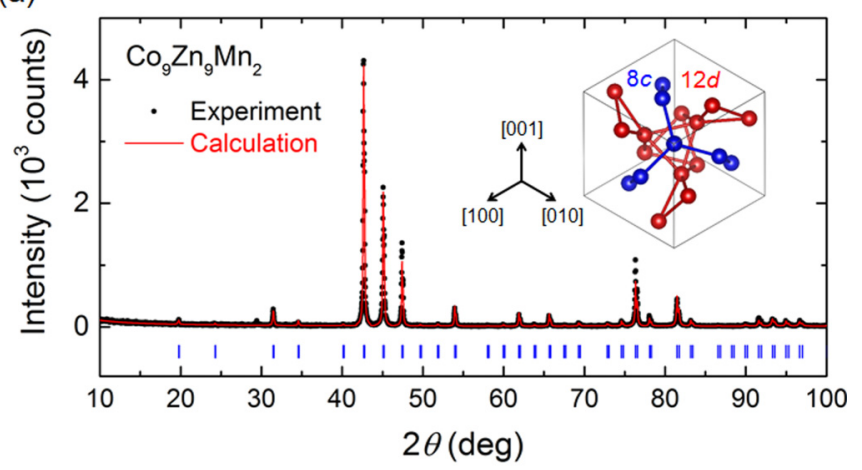

(b)

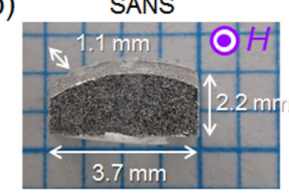

(c)

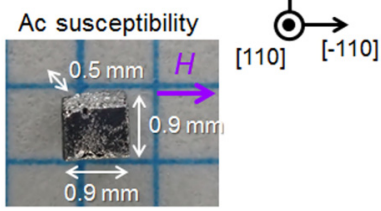

(d)

FIG. 2. (a) Powder x-ray diffraction profile for the sample of $\mathrm{Co}_{9} \mathrm{Zn}_{9} \mathrm{Mn}_{2}$. Experimental data (black circles) are compared with the simulation (red line) with a $\beta$-Mn-type structure, as illustrated in the inset, with a lattice parameter of $6.3578 \AA$. Peak positions are also indicated by blue bars. In $\mathrm{Co}_{9} \mathrm{Zn}_{9} \mathrm{Mn}_{2}$, the $8 c$ sites (blue circles in the inset) are mainly occupied by $\mathrm{Co}$, and the $12 d$ sites (red circles in the inset) are randomly occupied by $\mathrm{Co}, \mathrm{Zn}$, and Mn. Pictures of single-crystalline bulk samples of $\mathrm{Co}_{9} \mathrm{Zn}_{9} \mathrm{Mn}_{2}$ used for (b) SANS and (c) ac susceptibility measurements. (d) Laue picture of the (110) plane in the single-crystalline sample. The indicated crystal axes are common for (b)-(d).

single crystals used in this study, as shown in Fig. 2(a). A single-crystalline grain was cut along the (110), $(-110)$, and (001) planes with rectangular shape for SANS [Fig. 2(b)] and ac susceptibility [Fig. 2(c)] measurements after the crystal orientations were determined by the Laue x-ray diffraction method [Fig. 2(d)]. For the LTEM measurement, a tiny thinplate sample with (110) face and a thickness of approximately $150 \mathrm{~nm}$ was prepared from a single-crystalline bulk sample by a focused ion beam (FIB) of Ga.

\section{B. SANS measurement}

SANS measurements were performed using the instrument SANS-I at the Paul Scherrer Institute, Switzerland. Hightemperature measurements above room temperature were performed using a bespoke oven stick designed for SANS experiments. The neutron beam was collimated over a length of $18 \mathrm{~m}$ before the sample. The scattered neutrons were detected by a two-dimensional (2D) position-sensitive multidetector located $20 \mathrm{~m}$ behind the sample. The neutron wavelength was selected to be $10 \AA$ with a $10 \%$ full width at half maximum (FWHM) spread. The mounted single-crystalline sample was installed into a horizontal field cryomagnet so that the field direction was parallel to the [110] direction. Maintaining the 
magnetic field $(H) \|$ [110] geometry, the rocking angle $(\omega)$ between the neutron beam and the field direction was scanned in $2^{\circ}$ steps around the $H \|$ beam $(\omega=0)$ configuration, by rotating ("rocking") the cryomagnet together with the sample around the vertical [001] direction [see Fig. 6(b)]. The observed widths of the peaks (the FWHM) in scattering intensity versus $\omega$ were always broader than $\sim 5^{\circ}$. Therefore, the displayed SANS images are obtained by summing multiple SANS measurements taken over the range $-16^{\circ} \leqslant \omega \leqslant 10^{\circ}$ in order to observe both the intensity and the positions of all of the Bragg spots in single images such as those presented in this paper. The scanning condition of temperature and field in the FC process is as follows: (i) degaussing at $420 \mathrm{~K}$, (ii) zero field cooling (ZFC) to $390 \mathrm{~K}$, (iii) applying a field of $0.04 \mathrm{~T}$, and (iv) FC to measurement temperatures with a cooling rate of $\sim-2.0 \mathrm{~K} \mathrm{~min}^{-1}$ across the phase boundary $(384 \mathrm{~K}$ ) of the equilibrium SkX phase. The measurement time at each stabilized temperature and field with the above rocking scan was about $30 \mathrm{~min}$.

\section{C. ac susceptibility measurement}

The ac susceptibility measurements were performed by a superconducting quantum interference device magnetometer (MPMS3, Quantum Design) equipped with an ac susceptibility measurement option. The static magnetic field and the ac excitation magnetic field $(1 \mathrm{Oe}, 193 \mathrm{~Hz})$ were applied along the $[-110]$ direction. Due to the difference in shape between the samples used in the ac susceptibility and the SANS measurements, their demagnetization factors are different. To correct for this difference so that a common absolute magnetic field scale is used throughout this paper, the field values for the ac susceptibility measurements are recalibrated as $H_{\mathrm{c}}=2.0 \mathrm{H}$ [see Fig. 3(c)]. For all the figures related to ac susceptibility, the calibrated value $H_{\mathrm{c}}$ is used with the notation of $H$. The scanning condition of temperature and field in the FC process is as follows: (i) degaussing at $400 \mathrm{~K}$, (ii) ZFC to $395 \mathrm{~K}$, (iii) applying a field of $0.04 \mathrm{~T}$, and (iv) $\mathrm{FC}$ to measurement temperatures with a cooling rate of $\sim-10 \mathrm{~K} \mathrm{~min}^{-1}$ across the phase boundary $(384 \mathrm{~K})$ of the equilibrium SkX phase. The magnetic field was swept with a rate of $0.02 \mathrm{~T} \mathrm{~s}^{-1}$ in $4 \mathrm{mT}$ steps. The measurement time for each field was about $23 \mathrm{~s}$.

\section{LTEM measurement}

LTEM measurements were performed with a transmission electron microscope (JEM-2100F) at an acceleration voltage of $200 \mathrm{kV}$. Temperatures above room temperature were controlled by using a single-tilt heating holder. The temperatures presented in the text correspond to the holder temperature and, therefore, may be slightly different from the actual sample temperatures by approximately $\pm 10 \mathrm{~K}$. A magnetic field was applied perpendicular to the (110) plate and its magnitude was controlled by tuning the electric current of the objective lens. Due to the thin-plate sample shape ( $\sim 150 \mathrm{~nm}$ thick), its demagnetization factor and thus magnetic field scale are larger than those in bulk samples. The lateral magnetization distribution was obtained by the transport-of-intensity equation analysis of the overfocused and underfocused LTEM images [27].
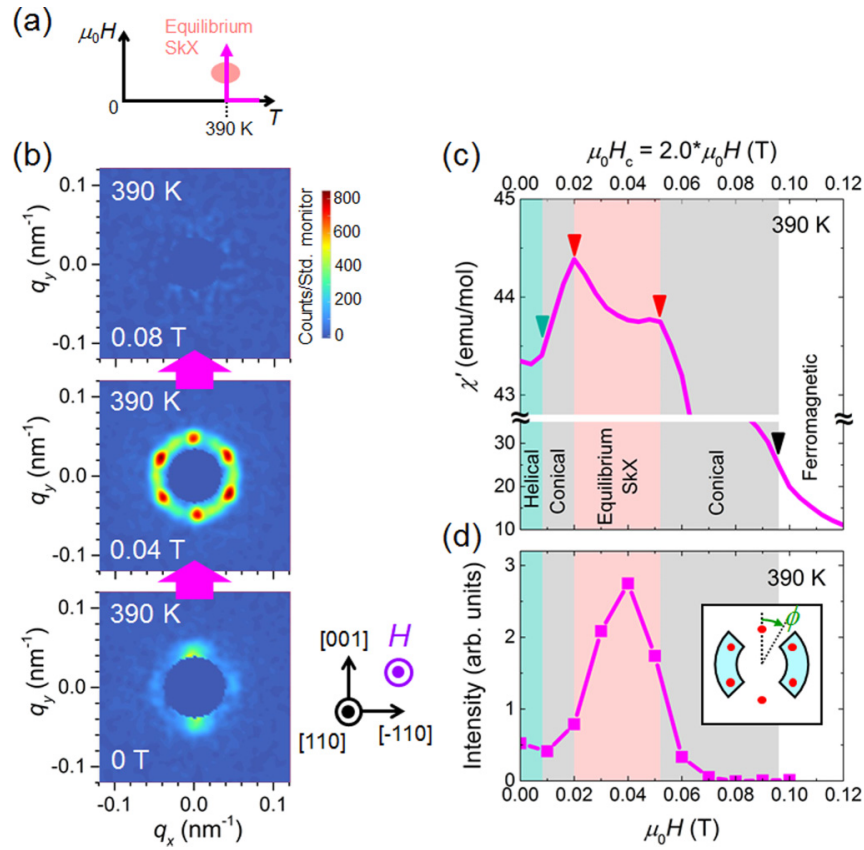

(d)

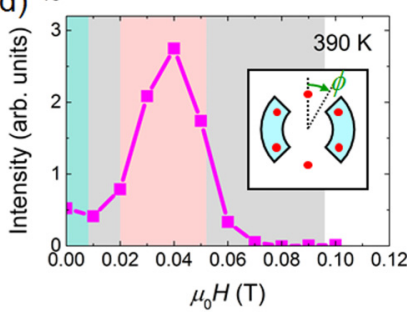

FIG. 3. (a) Schematic illustration of the measurement process. (b) SANS images at $390 \mathrm{~K}$ and selected fields. The intensity scale of the color plot is fixed for the three panels. (c) Field dependence of the real part of the ac susceptibility $\left(\chi^{\prime}\right)$. Since the demagnetization factor is different from that in the SANS measurement, the field values are calibrated as $H_{\mathrm{c}}=2.0 \mathrm{H}$. The phase boundary (dark green triangle) between the helical and conical states was determined as the inflection point of $\chi^{\prime}$. The phase boundaries (red triangles) between the equilibrium $\mathrm{SkX}$ and conical states were determined as the peak positions of $\chi^{\prime}$. The phase boundary (black triangle) between the conical and induced ferromagnetic states was determined as the inflection point of $\chi^{\prime}$. These boundaries and similar data points at different temperatures are plotted as solid symbols in the state diagram of Fig. 1(b). (d) Field dependence of the SANS intensity integrated over the azimuthal angle region at $\phi=90^{\circ} \pm 45^{\circ}$ and $270^{\circ} \pm 45^{\circ}$ (light blue region in the inset). Here, $\phi$ is defined as the clockwise azimuthal angle from the vertical [001] direction.

\section{RESULTS AND DISCUSSION}

\section{A. Identification of equilibrium SkX state}

First, we identified the equilibrium SkX state by SANS and ac susceptibility measurements in the field-sweeping process at $390 \mathrm{~K}$ after a ZFC (Fig. 3). As shown in the SANS images of Fig. 3(b), a helical state with two spots along the vertical [001] direction appears at $0 \mathrm{~T}$. With increasing magnetic field, a triangular-lattice SkX state with a hallmark six-spot pattern appears at $0.04 \mathrm{~T}$ as an equilibrium state. As shown in Fig. 3(d), the integrated SANS intensity originating from the equilibrium SkX shows large values in the range of $0.02 \leqslant \mu_{0} H \leqslant 0.05 \mathrm{~T}$, where the ac susceptibility shows a dip [Fig. 3(c)]. One of the triple $q$-vectors of the triangular-lattice SkX is aligned with the vertical [001] direction as in the case of $\mathrm{Co}_{8} \mathrm{Zn}_{8} \mathrm{Mn}_{4}[20,25]$. From the observed value of $q \sim 0.0485 \mathrm{~nm}^{-1}$ the lattice constant of the triangular-lattice SkX is calculated as $a_{\mathrm{sk}}=$ $(4 \pi / \sqrt{3}) q^{-1} \sim 150 \mathrm{~nm}$. Above $0.06 \mathrm{~T}$, the triangular-lattice SkX state changes to a conical state, in which the $q$-vector is parallel to the magnetic field, and no Bragg spot is observed 
in the present SANS configuration. Similar field-sweeping measurements for ac susceptibility were performed at different temperatures, and the phase boundaries of the equilibrium SkX state in the temperature $(T)-H$ plane were determined [red regions in the range $384 \leqslant T \leqslant 396 \mathrm{~K}$ in Fig. 1(b)].

\section{B. SANS measurements: Temperature sweep}

The temperature variation of the SANS patterns in a FC process $(0.04 \mathrm{~T})$ from 390 to $300 \mathrm{~K}$ and subsequent zero-field-warming (ZFW) process is shown in Fig. 4(b). In the FC process from the equilibrium $\mathrm{SkX}$ state, the six-spot pattern persists down to $300 \mathrm{~K}$ (although the spots become broader), clearly demonstrating the realization of the metastable SkX around room temperature. When, after the FC process, the magnetic field is decreased to zero while keeping the temperature at $300 \mathrm{~K}$, the six-spot pattern gradually changes to a four-spot pattern [see also Fig. 5(b)]. Here, the two horizontal spots are much broader than the two vertical spots, indicating that the broad horizontal spot is a superposition of the two original, now weaker, spots and a new, relatively stronger spot in between. As argued in the following, the broad four-spot pattern is assigned to a mixture of the original triangular-lattice SkX and a squarelike-lattice SkX, as illustrated in the left- and right-hand panels of Fig. 1(a), respectively. In the subsequent ZFW process from $300 \mathrm{~K}$, the four-spot pattern remains up to $345 \mathrm{~K}$ and finally changes to a two-spot pattern, similar to the bottom panel of Fig. 3(b) and hence corresponding to the equilibrium helical state at $390 \mathrm{~K}$.

For comparison, the temperature variation of the SANS images in a ZFC process from 390 to $300 \mathrm{~K}$ is shown in Fig. 4(c). The helical multidomain state with two stronger vertical spots and two much weaker horizontal spots was observed down to $300 \mathrm{~K}$. The relative intensities of the two horizontal spots to those of the two vertical spots are clearly distinct between the ZFC process [Fig. 4(c)] and the ZFW process [lower panels of Fig. 4(b)] after the FC via the equilibrium SkX phase.

Analysis of rocking scan data provides more detailed information on the scattering distribution of horizontal spots (Fig. 6). The temperature variation of the rocking curves (SANS intensity versus $\omega$ ) is shown in Fig. 6(c). In the FC process (blue triangles), the rocking curves show a maximum at $\omega=0$ down to $300 \mathrm{~K}$ since $q$-vectors of the metastable triangular-lattice SkX state lie close to the (110) plane and thus perpendicular to the field. In the subsequent ZFW process (red squares), the rocking curves also show peaks around $\omega \sim 0$ up to $375 \mathrm{~K}$ although the intensities are slightly lower than those in the FC process, demonstrating that horizontal $q$-vectors of the SANS images remain parallel to the (110) plane, as expected for a SkX state. On the other hand, the rocking curves in the ZFC process (green circles) show a broad minimum around $\omega \sim 0$ down to $300 \mathrm{~K}$ since the $q$-vectors of two of the helical multidomains are aligned with the [100] and [010] directions that are out of the (110) plane (and beyond the accessible range in $\omega)$. Thus, the two broad horizontal spots of the four-spot pattern observed in the ZFW process after the FC process arise not from a helical multidomain state with a $q$-vector aligned with the [100] or [010] direction. The increased intensity at the horizontal positions is also distinct from what is expected

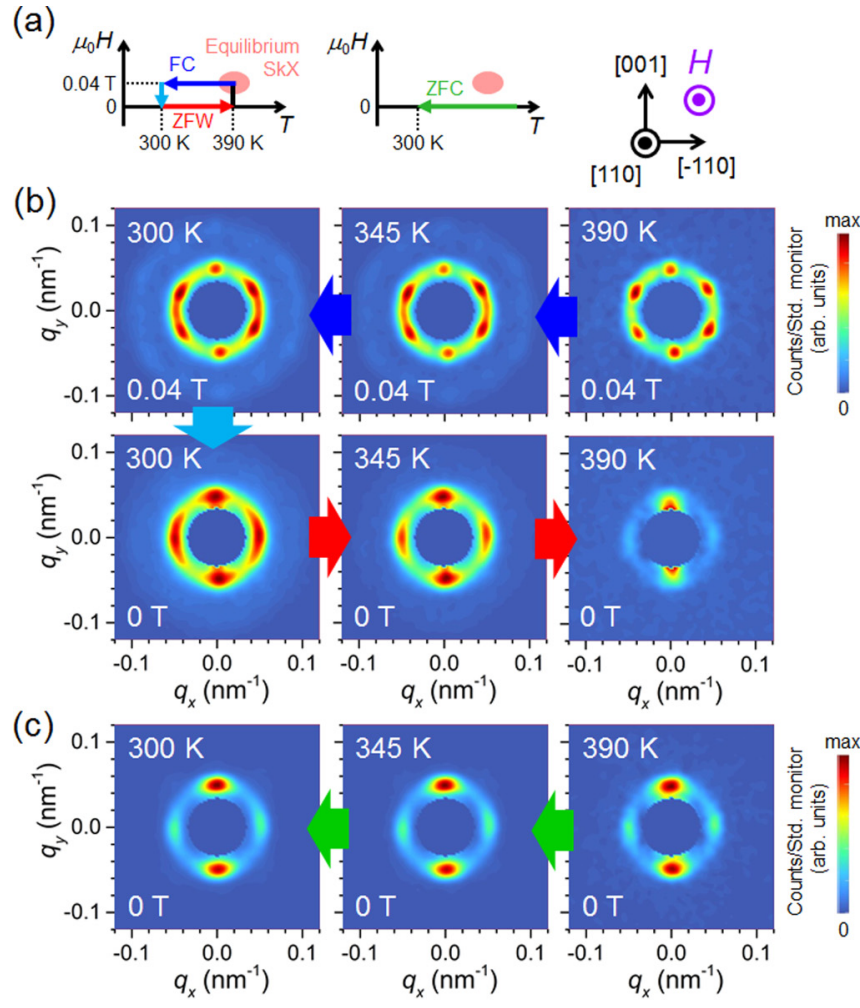

(d)

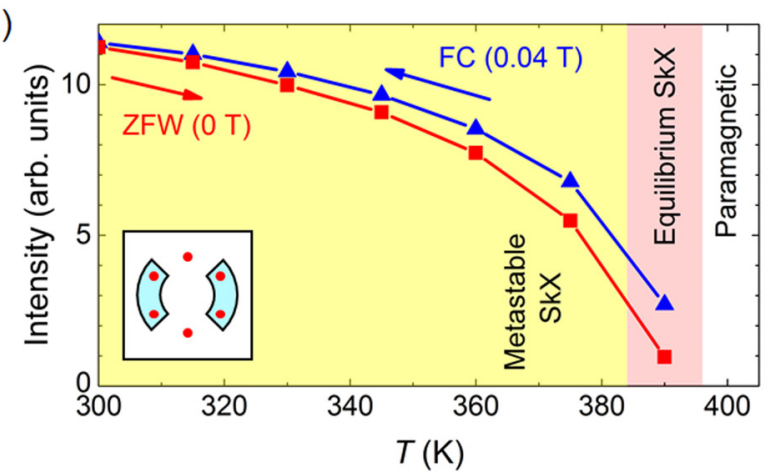

FIG. 4. (a) Schematic illustrations of the measurement processes. The colors of the arrows correspond to the colors of arrows in (b) and (c), and data points in (d). (b) SANS images in the FC $(0.04 \mathrm{~T})$ process and in the subsequent zero-field-warming (ZFW) process. The intensity scale of the color plots varies between each panel. (c) SANS images in the ZFC process. The intensity scale of the color plot varies between each panel. (d) Temperature dependence of the SANS intensity for the FC and ZFW processes, integrated over the same azimuthal angle area as for Fig. 3(d) (light blue region in the inset).

for the other helical state (or partially merged skyrmions as reported in $\mathrm{Fe}_{1-x} \mathrm{Co}_{x} \mathrm{Si}$ [16]) with a preferred $q$-vector aligned with the vertical [001] direction (as found at $390 \mathrm{~K}, 0 \mathrm{~T}$ ). Therefore, we conclude that the broad four-spot pattern at zero field after the FC and in the subsequent ZFW process represents a mixture of the original triangular-lattice SkX and a squarelike-lattice $\mathrm{SkX}$, as already mentioned.

The temperature dependence of the SANS intensity originating from the metastable SkX is displayed in Fig. 4(d), 
(a)
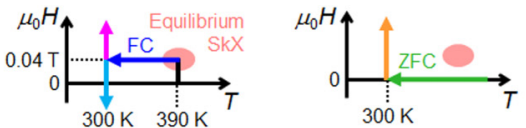

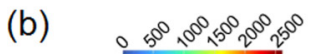
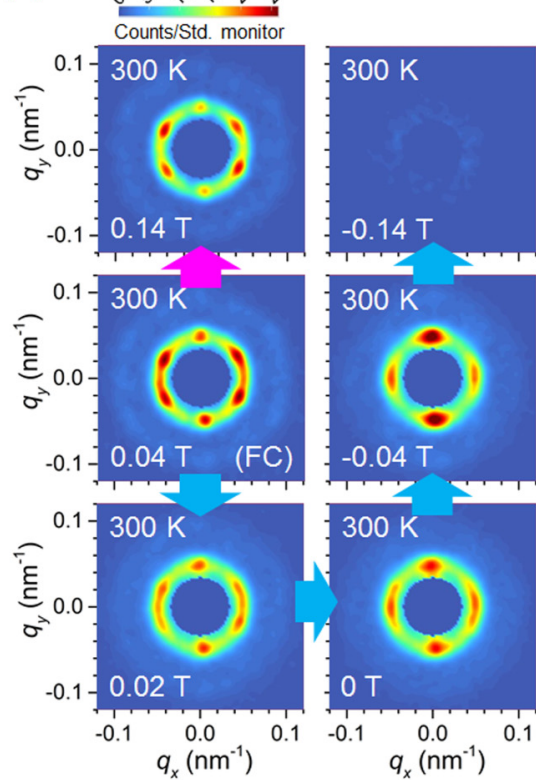

(c)

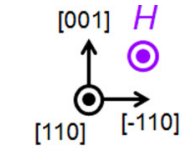

(d)

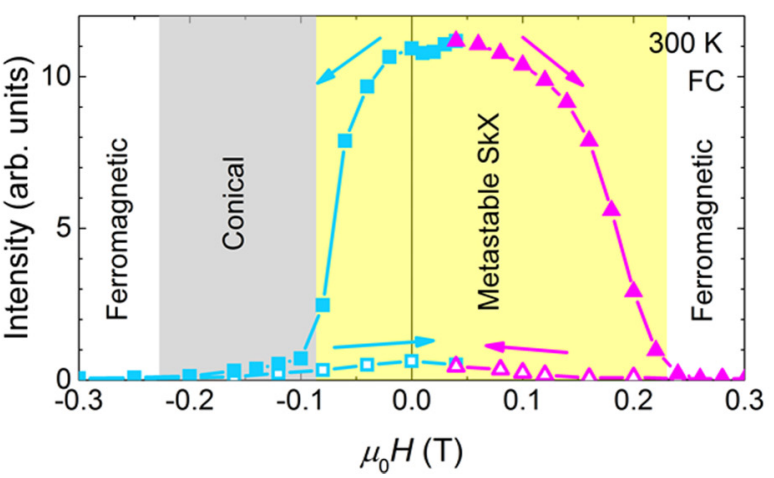

FIG. 5. (a) Schematic illustrations of the measurement processes. The colors of the arrows correspond to the colors of arrows in (b) and (c), and data points in (d). (b) SANS images at $300 \mathrm{~K}$ and selected magnetic fields in the field-sweeping processes after the FC. The intensity scale of the color plot is fixed for the six panels. (c) SANS images at $300 \mathrm{~K}$ and selected magnetic fields in the field-sweeping process after the ZFC. The intensity scale of the color plot is fixed for the three panels. (d) Field dependence of the SANS intensity at $300 \mathrm{~K}$ after the FC, integrated over the same azimuthal angle area as for Fig. 3(d). The intensities in field-sweeping runs from 0.04 to $\pm 0.3 \mathrm{~T}$ and the subsequent returning runs from $\pm 0.3 \mathrm{~T}$ back to $0.04 \mathrm{~T}$ are denoted by solid and open symbols with the same colors, respectively.

clearly indicating that the once-created metastable SkX survives up to $375 \mathrm{~K}$ at zero field.

\section{SANS measurements: Field sweep}

The field variation of SANS images at $300 \mathrm{~K}$ after a FC at $0.04 \mathrm{~T}$ is shown in Fig. 5(b). In the positive field direction, the six-spot pattern originating from the triangular-lattice SkX (a)
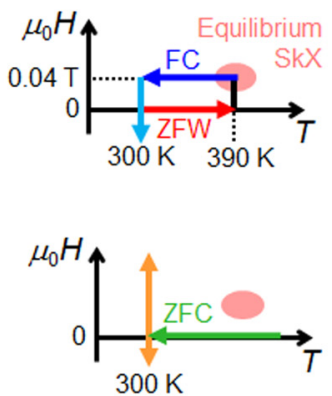

(c)

(b)

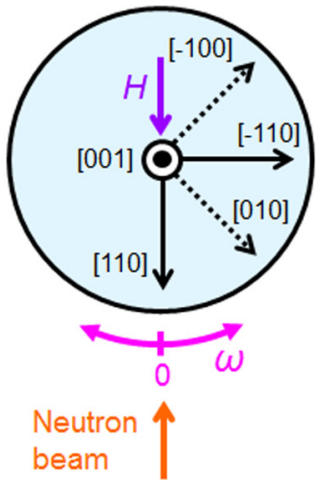

(d)
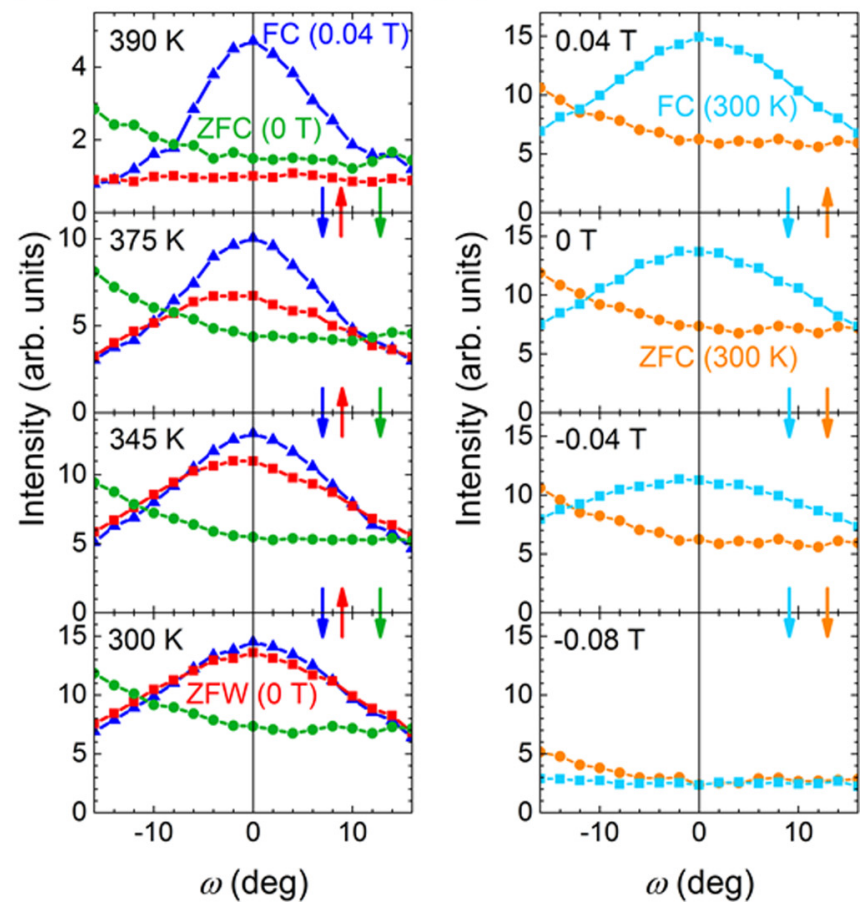

FIG. 6. (a) Schematic illustrations of the measurement processes. The colors of the arrows correspond to the colors of data points in (c) and (d). (b) Schematic view (from the vertical [001] direction) of the experimental configuration. Maintaining the $H \|$ [110] geometry, the angle $\omega$ between the neutron beam and the field direction is varied ("rocked") around the vertical [001] direction. The origin of the rocking angle ( $\omega=0)$ corresponds to the $q$-vector $\|$ (110) plane. For rocking curves (intensity versus $\omega$ ) in (c) and (d), SANS intensities are integrated over the same azimuthal angle area as for Fig. 3(d). (c) Rocking curves at selected temperatures for the FC process from 390 to $300 \mathrm{~K}$ (blue triangles), the subsequent ZFW process from 300 to $390 \mathrm{~K}$ (red squares), and the ZFC process (green circles). (d) Rocking curves at selected fields for the field sweeping from 0.04 to $-0.08 \mathrm{~T}$ after the FC (light blue squares), and the field sweeping from 0 to $0.04 \mathrm{~T}$ and to $-0.08 \mathrm{~T}$ after the ZFC (orange circles).

persists up to $0.14 \mathrm{~T}$. In the negative field direction, the six-spot pattern changes gradually to a four-spot pattern with broad side spots, indicating the gradual transformation of the SkX from the triangular lattice to a squarelike lattice with a broad region of coexistence. Upon further sweeping of the field in 
(a)

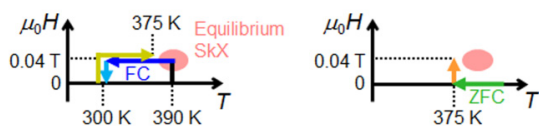

(b)

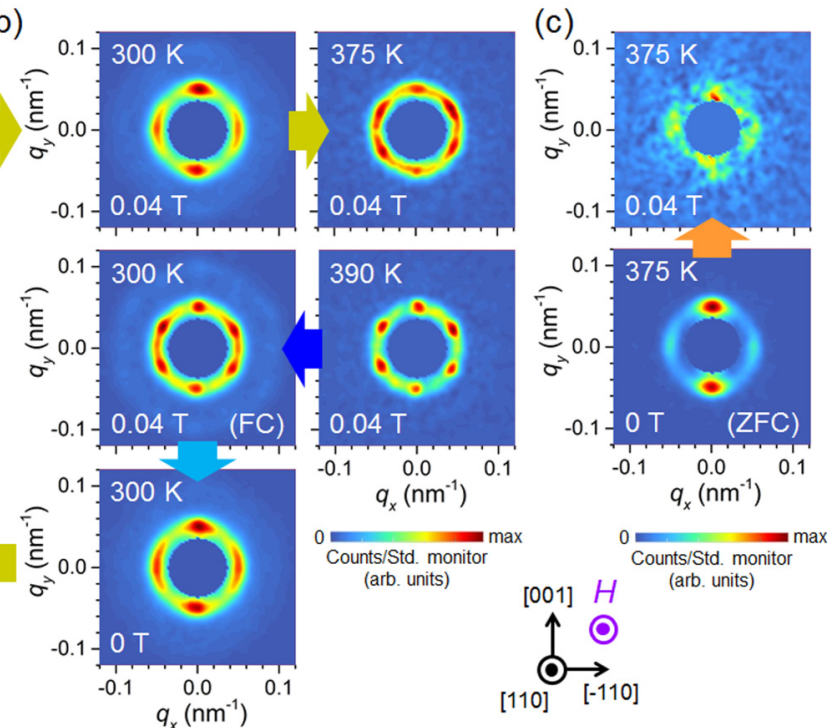

FIG. 7. (a) Schematic illustrations of the measurement processes. The colors of the arrows correspond to the colors of arrows in (b) and (c). (b) SANS images at selected temperatures and magnetic fields in the FC $(0.04 \mathrm{~T})$ to $300 \mathrm{~K}$, the subsequent field sweep to zero field, and the subsequent returning processes back to $0.04 \mathrm{~T}$ and $375 \mathrm{~K}$. The intensity scale of the color plot varies among the five panels. (c) SANS images at $375 \mathrm{~K}$ and at 0 and $0.04 \mathrm{~T}$ in the field-sweeping process after a ZFC. The intensity scale of the color plot varies between the two panels.

the negative direction, the metastable SkX changes to a conical state and no Bragg spot is observed at $-0.14 \mathrm{~T}$.

In the field sweeping at $300 \mathrm{~K}$ after a ZFC [Fig. 5(c)], the helical multidomain state with the two stronger vertical spots and two much weaker horizontal spots remains at $0.04 \mathrm{~T}$ and changes to a conical state at $0.14 \mathrm{~T}$ without any trace of $\mathrm{SkX}$ states.

Rocking curves in these field-sweeping measurements are shown in Fig. 6(d). In the field sweeping to negative fields after the FC (light blue squares), the peak structure of the rocking curves is preserved down to $-0.04 \mathrm{~T}$. These are also different from the valley-shaped rocking curves in the field sweeping after the ZFC process (orange circles), demonstrating the survival of the metastable SkX down to negative fields.

The field dependence of the SANS intensity originating from the metastable SkX is displayed in Fig. 5(d). This figure clearly indicates that the metastable $\mathrm{SkX}$ at $300 \mathrm{~K}$ survives over a very wide magnetic field region up to the conicalferromagnetic phase boundary and even down to the negative field region.

\section{SANS measurements: Returning process}

We have also investigated the reversibility of the transformation of the SkX. Figure 7(b) shows the temperature and field variations of the SANS images obtained in the returning process [yellow arrow in Fig. 7(a)] after the FC to $300 \mathrm{~K}$ and the subsequent field sweeping to zero field. When the magnetic field is returned back to $0.04 \mathrm{~T}$ at $300 \mathrm{~K}$, the broad four-spot pattern (coexistence of a triangular-lattice $\mathrm{SkX}$ and a squarelike-lattice SkX) persists. After increasing the temperature while keeping the magnetic field at $0.04 \mathrm{~T}$, the broad four-spot pattern survives up to higher temperatures and finally changes to a broad six-spot pattern (triangular-lattice $\mathrm{SkX}$ ) at $375 \mathrm{~K}$. It is noted that $375 \mathrm{~K}$ is well below the temperature range of the equilibrium SkX phase $(384 \leqslant T \leqslant$ $396 \mathrm{~K}$ ) as confirmed by the field sweeping at $375 \mathrm{~K}$ after a ZFC, in which no trace of SkX states was observed [Fig. 7(c)]. Therefore, the metastable SkX shows a reversible structural transition between a triangular lattice and a squarelike lattice with a broad coexistence region and a large hysteresis in the above temperature and field-sweeping procedures after the FC. This provides unambiguous evidence that indeed metastable skyrmions survive at $300 \mathrm{~K}$ and zero field.

The transition to a square-lattice SkX at low magnetic fields in the metastable state has been also observed in $\mathrm{Co}_{8} \mathrm{Zn}_{8} \mathrm{Mn}_{4}$ [25] and $\mathrm{MnSi}$ [28]. Theoretically, increased easy-plane anisotropy causes a stronger overlap of skyrmions, leading to a transition to the square-lattice SkX [29]. A similar situation emerges for the metastable $\mathrm{SkX}$ at zero external field; lowering the external field, instead of increasing easy-plane anisotropy, reduces the peripheral regions with upward moments to decrease the total magnetization.

\section{E. ac susceptibility measurements: Field sweep}

Figure 8(b) shows the field dependence of ac susceptibility at $300 \mathrm{~K}$ after several cooling processes. A large and asymmetric (with respect to the sign of the field) hysteresis is observed between -0.09 and $0.23 \mathrm{~T}$ after a $\mathrm{FC}$ process (FC1) via the equilibrium SkX phase. On the other hand, the ZFC process and another FC process (FC2) that bypasses the equilibrium SkX phase show smaller and symmetric hystereses that are related to changes between multidomain helical states and the conical state. These indicate that the asymmetric hysteresis is observed only after $\mathrm{FC} 1$ stems from the supercooled metastable $\mathrm{SkX}$, which is totally consistent with the asymmetry in the field dependence of the SANS intensity for the metastable SkX shown in Fig. 8(c). Similar field sweepings after a FC via the equilibrium SkX phase were performed at several temperatures [Fig. 8(d)], and the boundary of the metastable SkX state in the $T-H$ plane was determined [yellow region in Fig. 1(b)], clearly indicating the realization of the metastable SkX in a wide temperature and magnetic field region including room temperature and zero magnetic field.

\section{F. ac susceptibility measurements: Temporal dependence}

Figure 9(a) shows the temporal dependence of the normalized ac susceptibility at several temperatures after a FC via the equilibrium SkX phase. The observed relaxation time from the metastable SkX to the equilibrium conical state (lifetime of the metastable $\mathrm{SkX}$ ) is quite long even at $380 \mathrm{~K}$, just below the equilibrium SkX phase boundary. The relaxation time further increases by lowering temperature and finally the relaxation behavior is hardly observable at $350 \mathrm{~K}$. 
(a)
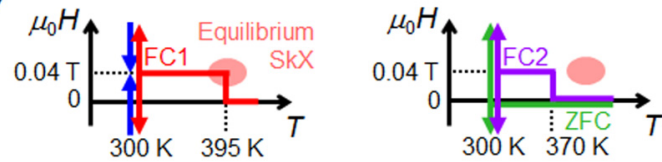

(b)

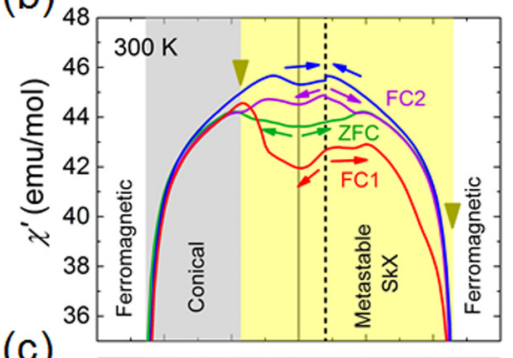

(c)

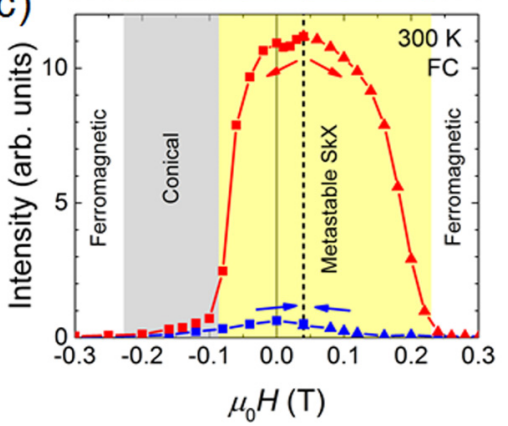

(d)

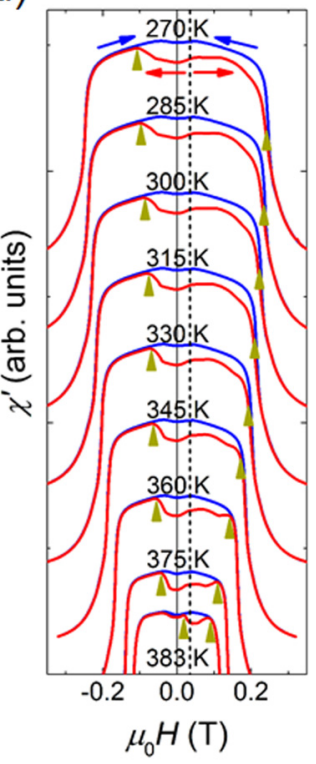

FIG. 8. (a) Schematic illustrations of the measurement processes. The colors of the arrows correspond to the colors of data lines in (b). (b) Field dependence of the real part of the ac susceptibility $\left(\chi^{\prime}\right)$ at $300 \mathrm{~K}$ after several cooling processes shown in (a). The results of the field sweepings (not shown) from $\pm 0.3 \mathrm{~T}$ back to $0.04 \mathrm{~T}(0 \mathrm{~T})$ after FC2 (ZFC) are almost the same as that after FC1. The yellow triangles denote the boundaries of the metastable SkX state. The boundary at the positive field side is determined as the field where the hysteresis of $\chi^{\prime}$ (red and blue lines) closes, and the boundary at the negative field side is determined as the field where $\chi^{\prime}$ (red line) shows a peak. (c) Field dependence of the SANS intensity for metastable SkX at $300 \mathrm{~K}$ after the FC [the same data as in Fig. 5(d)] is shown again for the purpose of comparison with (b). (d) Field dependencies of $\chi^{\prime}$ at several different temperatures after the FC process via the equilibrium state. The yellow triangles denote the boundaries of the metastable SkX that are plotted in the state diagram in Fig. 1(b).

In order to quantitatively discuss the robust metastability, the relaxation time $\tau$, estimated from the fits to stretched exponential functions, is plotted against temperature and fitted to a modified Arrhenius law [30] in Fig. 9(b). The activation energy of the decay rate $\tau^{-1}$ reaches as high as $1.0 \times 10^{4} \mathrm{~K}$ even at $350 \mathrm{~K}$, well above room temperature. Remarkably, this protection energy scale for the metastable skyrmions is much higher than the other magnetic interaction energies, such as the ferromagnetic interaction and the DMI (several hundred kelvin and several kelvin, respectively, in the present case), and hence perhaps attributed to the topological nature of the present skyrmions that display a slow spatial variation of the spin orientation [31]. The obtained coefficient of relaxation time $\tau_{0} \sim 47 \mathrm{~s}$ is five orders of magnitude longer than that in $\mathrm{MnSi}$ $\left(\tau_{0} \sim 2.7 \times 10^{-4} \mathrm{~s}\right)$ [30]. Since the critical cooling rate for quenching an equilibrium phase to lower temperatures is in- (a)

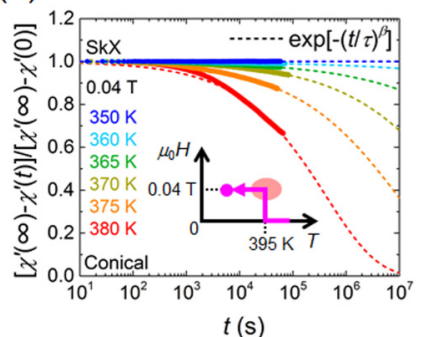

(b)

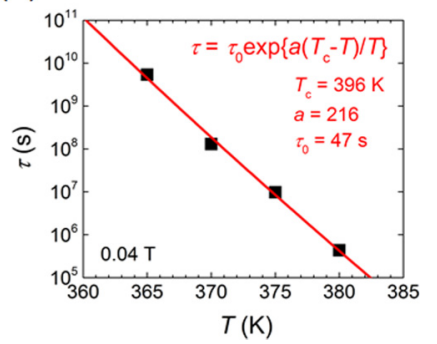

FIG. 9. (a) Temporal dependence of the normalized $\chi^{\prime}(t)$ at $0.04 \mathrm{~T}$ and several temperatures after a FC process via the equilibrium state (as schematically depicted in the inset). $\chi^{\prime}(0)$ is an initial value (metastable SkX state), and $\chi^{\prime}(\infty)$ is a fully relaxed value (equilibrium conical state) which is assumed to be the value of $\chi^{\prime}$ at $0.04 \mathrm{~T}$ in the field-decreasing run from $0.3 \mathrm{~T}$. The data points are fitted to stretched exponential functions (dotted lines), $\exp \left\{-(t / \tau)^{\beta}\right\}$. The obtained $\beta$ values are in the range of 0.3 to 0.5 , indicating a highly inhomogeneous distribution of relaxation times. (b) Temperature dependence of the relaxation time $\tau$ determined by the fits in (a). The experimental data (black symbols) are fitted to a modified Arrhenius law (red line), $\tau=\tau_{0} \exp \left\{a\left(T_{\mathrm{c}}-T\right) / T\right\}$, with an assumption of a temperature-dependent activation energy [30]. The obtained parameters are $a=216$ and $\tau_{0}=47 \mathrm{~s}$.

versely correlated to $\tau_{0}$, the moderately slow cooling rate is sufficient to quench the equilibrium SkX state in the present case.

Plausible origins of the long lifetime of metastable skyrmions in $\mathrm{Co}_{9} \mathrm{Zn}_{9} \mathrm{Mn}_{2}$ are random site occupancies [23] (the $8 c$ site is mainly occupied by Co while the $12 d$ site is occupied by a random mixture of $\mathrm{Co}, \mathrm{Zn}$, and $\mathrm{Mn}$ ) and a larger skyrmion size (i.e., the number of spins involved in one skyrmion) of $150 \mathrm{~nm}$ as compared to $18 \mathrm{~nm}$ in MnSi. Since the skyrmion size is much larger than the distance between nearest-neighbor spins (on the order of several angstroms), the random site occupancies give rise to "weak pinning" in the terminology of density wave physics [34], which may play an important role in the robust metastability of each skyrmion. A similar robust metastable $\mathrm{SkX}$ that is accessible by a moderate FC and can survive at zero field has been reported in $\mathrm{Fe}_{1-x} \mathrm{Co}_{x} \mathrm{Si}$ alloys $[16,35]$ with random site occupancy and a relatively large skyrmion size (90 $\mathrm{nm}$ at $x=0.5)$, in which the origin may be the same as in the present case.

\section{G. LTEM measurements}

We further confirmed the formation of skyrmions by realspace observations using LTEM on a (110) thin-plate sample with a thickness of $\sim 150 \mathrm{~nm}$. As shown in Fig. 10(b), we observed the triangular-lattice SkX state at approximately $370 \mathrm{~K}$ and $0.07 \mathrm{~T}$ as the equilibrium state. The SkX state persists down to $290 \mathrm{~K}$ as the metastable state in a FC process. The metastable SkX at $290 \mathrm{~K}$ is barely influenced upon decreasing the magnetic field and remains triangularly coordinated at zero magnetic field. The characteristic swirling of the magnetic moments in a skyrmion is clearly shown by the angular color coding in Fig. 10(c) deduced by a transport-of-intensity equation analysis [27]. In the subsequent $\mathrm{ZFW}$ process, the triangular-lattice $\mathrm{SkX}$ survives up to at least $350 \mathrm{~K}$ and finally changes to the equilibrium helical state at $370 \mathrm{~K}$. 
(a)

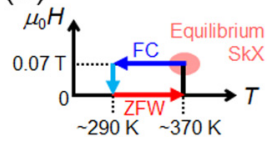

(b)

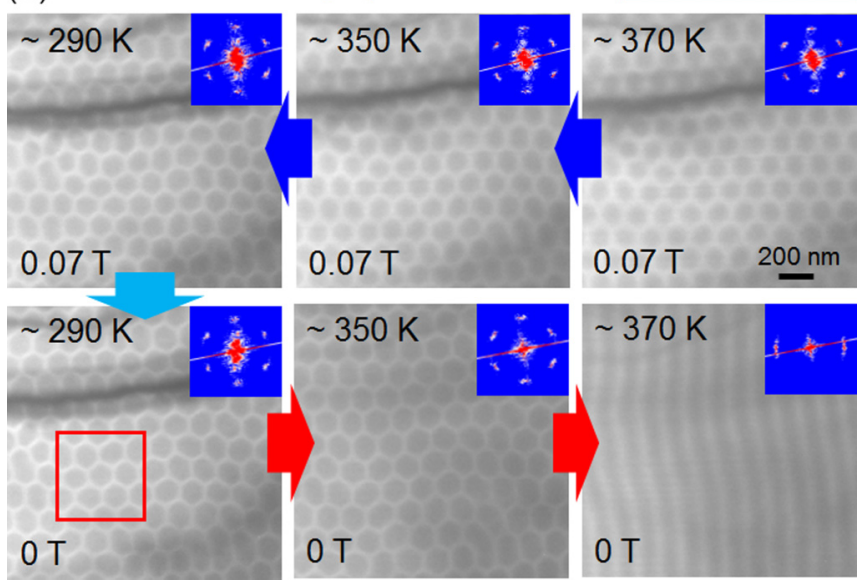

FIG. 10. (a) Schematic illustration of the measurement process. The colors of the arrows correspond to the colors of arrows in (b). (b) Overfocused LTEM images on the (110) plane in the FC process and in the subsequent ZFW process. The defocus values of LTEM images were set to be $+288 \mu \mathrm{m}$ at $370 \mathrm{~K},+192 \mu \mathrm{m}$ at $350 \mathrm{~K}$, and +96 $\mu \mathrm{m}$ at $290 \mathrm{~K}$, respectively. These LTEM images were taken for nearly the same sample position. The insets of the respective panels show the fast Fourier-transformed patterns of the LTEM images taken over a wider sample area. (c) Distribution of in-plane magnetic moments for the area indicated by the red square in (b) at $290 \mathrm{~K}$ and zero magnetic field after the FC, as deduced from a transport-of-intensity equation analysis of overfocused and underfocused LTEM images. The color wheel represents the direction and magnitude of the in-plane magnetization.

The field variation of LTEM images in the negative direction at $290 \mathrm{~K}$ after the $\mathrm{FC}$ and the subsequent sample reversal procedure is shown in Fig. 11(b). Upon increasing the magnitude of the field in the negative direction, although some skyrmions merge with each other and the total topological charge decreases, a number of skyrmions remain and keep the original triangular lattice at $-0.049 \mathrm{~T}$, clearly demonstrating the survival of a significant metastable skyrmion density in a negative field region. The observed positional dependence may arise from inhomogeneity of effective magnetic fields (due to a demagnetization effect) or random pinning for the spin textures. Upon further increasing the field in the negative direction, the number of skyrmions that merge with each other increases while some topological structures still remain at $-0.078 \mathrm{~T}$, and finally almost all the skyrmions are destroyed and change to a helical state.

In comparison with the SANS result, while the metastable SkX is commonly observed irrespective of the sample thickness, both the lattice forms of skyrmions at zero field and the preferred $q$-vector alignments of helical states are different between bulk and thin-plate samples. These differences arise presumably due to the differences in the sample shapes. The dipole interaction between skyrmion tubes working as an (a)

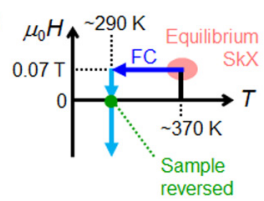

(b)
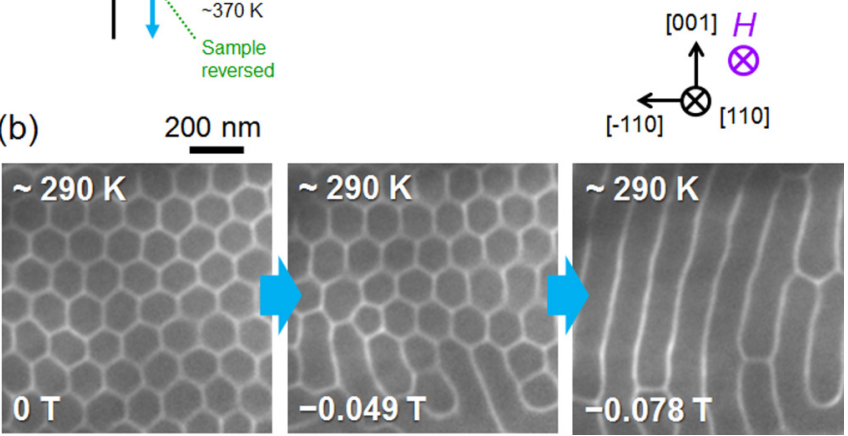

FIG. 11. (a) Schematic illustration of the measurement process. After the FC via the equilibrium SkX phase to $290 \mathrm{~K}$ and the subsequent field-decreasing process to zero field, the sample was taken out of the electron microscope, turned upside down, and installed into the electron microscope again. Subsequently, the magnetic field was applied along the same (positive) direction for the electron microscope as before, which was the opposite (negative) direction for the sample. (b) Underfocused LTEM images on the (110) plane at $290 \mathrm{~K}$ and selected magnetic fields in the field-sweeping process from zero field to negative fields, and after the above sample reversing procedure. The defocus values of the LTEM images were set to be $-288 \mu \mathrm{m}$ for 0 and $-0.049 \mathrm{~T}$, and $-384 \mu \mathrm{m}$ for $-0.078 \mathrm{~T}$, respectively.

effective repulsive interskyrmion force is significant only in the case of thin-plate samples, and it dominates over the shape anisotropy effect toward in-plane magnetization, tending to preserve the close-packed triangular lattice, and thereby preventing the SkX lattice transformation in the case of the thin-plate sample. Magnetocrystalline anisotropy tends to favor magnetic moment directions along [100] and its equivalent [24], thus giving rise to the preferred $q$-vector direction along [001] in the bulk. For the case of the (110) thin-plate sample, shape anisotropy towards the in-plane magnetization coincides with the magnetocrystalline anisotropy for $q \|[-110]$, but not for $q \|$ [001], thus leading to $q \|[-110]$ as observed in Fig. 10(b).

\section{CONCLUSION}

We have demonstrated the realization of highly robust metastable skyrmions in a wide temperature and field range, including room temperature and zero magnetic field, in $\mathrm{Co}_{9} \mathrm{Zn}_{9} \mathrm{Mn}_{2}$ by field cooling from an equilibrium skyrmion phase. The robustness of skyrmions in such a wide parameter space appears characteristic of topological spin textures accompanied by both quenched random disorder and the slowly varying nature of the magnetic moment orientation within a single skyrmion. The robust metastable skyrmions arising from the Dzyaloshinskii-Moriya interaction associated with the chiral structure of the bulk thus present as highly suitable for both investigating the fundamental properties of topological spin textures, and also practical applications of skyrmions. 


\section{ACKNOWLEDGMENTS}

We are grateful to N. Nagaosa, W. Koshibae, X. Z. Yu, F. Kagawa, T. Nakajima, and H. Furukawa for fruitful discussions. This work was supported by the Japan Society for the Promotion of Science (JSPS) Grant-in-Aids for Scientific
Research (S) No. 24224009 and for Young Scientists (B) No. 17K18355, the Swiss National Science Foundation (SNSF) Sinergia network "NanoSkyrmionics" (Grant No. CRSII5171003), SNSF Projects No. 153451 and No. 166298, and the European Research Council project CONQUEST.

K.K. and J.S.W. contributed equally to this work.
[1] A. N. Bogdanov and D. A. Yablonskii, Sov. Phys. JETP 68, 101 (1989).

[2] N. Nagaosa and Y. Tokura, Nat. Nanotechnol. 8, 899 (2013).

[3] S. Mühlbauer, B. Binz, F. Jonietz, C. Pfleiderer, A. Rosch, A. Neubauer, R. Georgii, and P. Böni, Science 323, 915 (2009).

[4] X. Z. Yu, Y. Onose, N. Kanazawa, J. H. Park, J. H. Han, Y. Matsui, N. Nagaosa, and Y. Tokura, Nature (London) 465, 901 (2010).

[5] F. Jonietz, S. Muhlbauer, C. Pfleiderer, A. Neubauer, W. Münzer, A. Bauer, T. Adams, R. Georgii, P. Böni, R. A. Duine, K. Everschor, M. Garst, and A. Rosch, Science 330, 1648 (2010).

[6] T. Schulz, R. Ritz, A. Bauer, M. Halder, M. Wagner, C. Franz, C. Pfleiderer, K. Everschor, M. Garst, and A. Rosch, Nat. Phys. 8, 301 (2012).

[7] X. Z. Yu, N. Kanazawa, W. Z. Zhang, T. Nagai, T. Hara, K. Kimoto, Y. Matsui, Y. Onose, and Y. Tokura, Nat. Commun. 3, 988 (2012).

[8] J. Iwasaki, M. Mochizuki, and N. Nagaosa, Nat. Nanotechnol. 8, 742 (2013).

[9] J. Sampaio, V. Cros, S. Rohart, A. Thiaville, and A. Fert, Nat. Nanotechnol. 8, 839 (2013).

[10] S. Heinze, K. von Bergmann, M. Menzel, J. Brede, A. Kubetzka, R. Wiesendanger, G. Bihlmayer, and S. Blügel, Nat. Phys. 7, 713 (2011).

[11] N. Romming, C. Hanneken, M. Menzel, J. E. Bickel, B. Wolter, K. Bergmann, A. Kubetzka, and R. Wiesendanger, Science 341, 636 (2013)

[12] O. Boulle, J. Vogel, H. Yang, S. Pizzini, D. de S. Chaves, A. Locatelli, T. O. Menteş, A. Sala, L. D. Buda-Prejbeanu, O. Klein, M. Belmeguenai, Y. Roussigné, A. Stashkevich, S. M. Chérif, L. Aballe, M. Foerster, M. Chshiev, S. Auffret, I. M. Miron, and G. Gaudin, Nat. Nanotechnol. 11, 449 (2016).

[13] S. Woo, K. Litzius, B. Krüger, M.-Y. Im, L. Caretta, K. Richter, M. Mann, A. Krone, R. Reeve, M. Weigand, P. Agrawal, P. Ischer, M. Kläui, G. S. D. Beach, I. Lemesh, and M.-A. Mawass, Nat. Mater. 15, 501 (2016).

[14] S. Ishiwata, M. Tokunaga, Y. Kaneko, D. Okuyama, Y. Tokunaga, S. Wakimoto, K. Kakurai, T. Arima, Y. Taguchi, and Y. Tokura, Phys. Rev. B 84, 054427 (2011).

[15] I. Kézsmárki, S. Bordács, P. Milde, E. Neuber, L. M. Eng, J. S. White, H. M. Rønnow, C. D. Dewhurst, M. Mochizuki, K. Yanai, H. D. Ehlers, V. Tsurkan, and A. Loidl, Nat. Mater. 14, 1116 (2015)

[16] P. Milde, D. Köhler, J. Seidel, L. M. Eng, A. Bauer, A. Chacon, J. Kindervater, S. Mühlbauer, C. Pfleiderer, S. Buhrandt, C. Schütte, and A. Rosch, Science 340, 1076 (2013).
[17] X. Z. Yu, N. Kanazawa, Y. Onose, K. Kimoto, W. Z. Zhang, S. Ishiwata, Y. Matsui, and Y. Tokura, Nat. Mater. 10, 106 (2011).

[18] S. Seki, X. Z. Yu, S. Ishiwata, and Y. Tokura, Science 336, 198 (2012).

[19] J. S. White, K. Prša, P. Huang, A. A. Omrani, I. Živković, M. Bartkowiak, H. Berger, A. Magrez, J. L. Gavilano, G. Nagy, J. Zang, and H. M. Rønnow, Phys. Rev. Lett. 113, 107203 (2014).

[20] Y. Tokunaga, X. Z. Yu, J. S. White, H. M. Rønnow, D. Morikawa, Y. Taguchi, and Y. Tokura, Nat. Commun. 6, 7638 (2015).

[21] M. Bode, M. Heide, K. von Bergmann, P. Ferriani, S. Heinze, G. Bihlmayer, A. Kubetzka, O. Pietzsch, S. Blügel, and R. Wiesendanger, Nature (London) 447, 190 (2007).

[22] A. Hrabec, N. A. Porter, A. Wells, M. J. Benitez, G. Burnell, S. McVitie, D. McGrouther, T. A. Moore, and C. H. Marrows, Phys. Rev. B 90, 020402(R) (2014).

[23] T. Hori, H. Shiraish, and Y. Ishii, J. Magn. Magn. Mater. 310, 1820 (2007).

[24] W. Xie, S. Thimmaiah, J. Lamsal, J. Liu, T. W. Heitmann, D. Quirinale, A. I. Goldman, V. Pecharsky, and G. J. Miller, Inorg. Chem. 52, 9399 (2013).

[25] K. Karube, J. S. White, N. Reynolds, J. L. Gavilano, H. Oike, A. Kikkawa, F. Kagawa, Y. Tokunaga, H. M. Rønnow, Y. Tokura, and Y. Taguchi, Nat. Mater. 15, 1237 (2016).

[26] D. Morikawa, X. Z. Yu, K. Karube, Y. Tokunaga, Y. Taguchi, T. Arima, and Y. Tokura, Nano Lett. 17, 1637 (2017).

[27] K. Ishizuka and B. Allman, J. Electron Microsc. 54, 191 (2005).

[28] T. Nakajima, H. Oike, A. Kikkawa, E. P. Gilbert, N. Booth, K. Kakurai, Y. Taguchi, Y. Tokura, F. Kagawa, and T. Arima, Sci. Adv. 3, e1602562 (2017).

[29] S.-Z. Lin, A. Saxena, and C. D. Batista, Phys. Rev. B 91, 224407 (2015).

[30] H. Oike, A. Kikkawa, N. Kanazawa, Y. Taguchi, M. Kawasaki, Y. Tokura, and F. Kagawa, Nat. Phys. 12, 62 (2016).

[31] While it is suggested that topology plays a minor role in a small skyrmion with a size of several nm [32,33], the skyrmion in the present material has a relatively large diameter as compared with the distance between spins, and hence the spins are spatially gradually twisted, making the role of topology important.

[32] S. Rohart, J. Miltat, and A. Thiaville, Phys. Rev. B 93, 214412 (2016).

[33] D. Stosic, J. Mulkers, B. Van Waeyenberge, T. B. Ludermir, and M. V. Milošević, Phys. Rev. B 95, 214418 (2017).

[34] G. Grüner, Rev. Mod. Phys. 60, 1129 (1988).

[35] W. Münzer, A. Neubauer, T. Adams, S. Mühlbauer, C. Franz, F. Jonietz, R. Georgii, P. Böni, B. Pedersen, M. Schmidt, A. Rosch, and C. Pfleiderer, Phys. Rev. B 81, 041203(R) (2010). 